\title{
Rating Agencies Interoperation for Peer-to-Peer Online Transactions
}

\author{
Mihaela Ion*, Hristo Koshutanski ${ }^{\dagger}$, Volker Hoyer ${ }^{\ddagger}$ and Luigi Telesca* \\ ${ }^{*}$ CREATE-NET, Via alla Cascata 56/D, Povo-Trento, Italy \\ \{mihaela.ion, luigi.telesca\}@create-net.org \\ ${ }^{\dagger}$ Computer Science Department, University of Malaga, Spain \\ hristo@1cc.uma.es \\ $\ddagger$ University of St. Gallen, Switzerland \\ volker.hoyer@unisg.ch
}

\begin{abstract}
In current peer-to-peer systems users interact with unknown services and users for the purpose of online transactions such as file sharing and trading of commodities. Peer-to-Peer reputation systems allow users to assess the trustworthiness of unknown entities based on subjective feedback from the other peers. However, this cannot constitute sufficient proof for many transactions like service composition, negotiations and coalition formation in which users require more solid proof of the quality of unknown services. Ratings certified by trusted third parties in the form of a security token are objective and reliable and, hence, allow building trust between peers. Because of the decentralized and distributed nature of peer-to-peer networks, a central authority (or hierarchy of them) issuing such certificates would not scale up. We propose a framework for peer-to-peer agencies interoperation based on rating certificates and metacertificates describing bilateral agencies relations.
\end{abstract}

\section{INTRODUCTION}

A large number of peer-to-peer $(\mathrm{P} 2 \mathrm{P})$ reputation systems allow peers to compute a local or global trust value of unknown entities based on recommendations issued by other peers in the system. These values are subjective, sometimes depend on the peer computing them and many times can be manipulated by malicious peers in the network. Because of this, P2P reputation values are not suitable for many high-value online transactions, negotiations, service compositions or dynamic coallision formations. In order to facilitate such transactions, certified and standardized ratings of services are needed. Such rating certificates coming from trusted third parties would enable creating new trust relations and collaborations.

The idea of rating agencies is not new. Financial rating agencies exist for more than a century and lately many organizations issue certificates to software applications and Web sites based on different criteria as we shall examine later in the section. However, a model for decentralized rating agencies interoperation suitable for online transactions in $\mathrm{P} 2 \mathrm{P}$ networks is missing.

The paper proposes a framework for P2P distributed agencies that bootstraps trust relations between entities from different network domains. The framework aims at achieving onthe-fly interoperation between agencies through standardization of certificates and meta-certificates describing relations between agencies.
The paper is organized as following. Next, we overview trust management in $\mathrm{P} 2 \mathrm{P}$ systems and of agencies that come close to our envisioned model for rating agencies: financial rating agencies and organizations able to provide ratings for software applications and Web sites. Section II describes the new rating agencies model and provides its high-level functional description. Section III looks inside the interoperability notion and defines the core building blocks introduced in Section II. Section IV concludes the paper and outlines future work.

\section{A. Trust management in peer-to-peer systems}

There are several approaches for enabling trust in $\mathrm{P} 2 \mathrm{P}$ systems. In the following we discuss reputation systems, credential-based trust, and institutional trust.

1) $P 2 P$ reputation systems: $\mathrm{P} 2 \mathrm{P}$ reputation systems rely mainly on peer recommendations to compute a global [8], [15], [13], [5] or local [3], [10] reputation value for each peer in the system. The factors taken into consideration and the way the reputation values are computed differ from system to system. While transaction feedback issued by peers constitutes the main factor, the number, size and time of the transaction can also be taken into consideration together with the credibility of the peer making the recommendation. PeerTrust [14] goes even more general and computes a transaction context factor and a community context factor.

Auction sites like eBay ${ }^{1}$, Amazon $^{2}$, and Yahoo! Auction ${ }^{3}$ allow users to trade commodities online in a customer-tocustomer (C2C) fashion. To build customer trust, these sites rely on reputation systems which allow users to decide whom to trust, encourage trustworthy behavior, and help detect dishonest peers.

2) Credential-based trust: Distributed Public Key Infrastructure (PKI) can provide a higher level of security in P2P networks. In [4], the authors propose a completely decentralized P2P PKI which can serve as the basis for higher-level security services. The long-term vision of the authors is a $\mathrm{P} 2 \mathrm{P}$ ecommerce platform which addresses security (authentication, authorization, accountability, trust). The model allows proper

\footnotetext{
${ }^{1}$ http://www.ebay.com

${ }^{2}$ http://www.amazon.com

${ }^{3}$ http://auctions.yahoo.com
} 
peer identification, but trust is still computed by means of recommendations from peers which can be subjective.

3) Institutional trust: Institutional trust means that trust in one institution or organization can be extended to its employees. In [7] we extend the notion of institutional trust to social institutional trust and allow institutions to build social networks of trust based on their business relations. The trust relations an institution has with others are expressed in a metadata certificate. These certificates allow extending trust on an institution to the partners of that institution.

The large number of transactions taking place on auction sites (eBay has more than 4 million auctions open at a time) proves the success of reputation systems and the high demand for $\mathrm{C} 2 \mathrm{C}$ commerce. However, reputation systems have certain shortcomings which limit their applicability to low-value transactions. P2P reputation systems compute trust mainly based on peer recommendations which can be subjective and manipulated by malicious peers. Moreover, each peer uses its own evaluation criteria and interprets reputation values differently. Because of that, reputation values do not allow building a strong trust relation between unknown peers that want to engage in a high-value transaction. We argue that certified ratings based on clearly defined criteria which is evaluated by a trusted third party overcome the above mentioned shortcomings of P2P reputation systems. Our model uses certified ratings together with social institutional trust to allow building trust between peers in an objective and distributed manner. Because of that, the model we propose could be used for commercial scenarios with bigger constraints.

\section{B. Online transactions in dynamic and decentralized $P 2 P$ environments}

Auction sites provide a centralized trading platform in which one central authority controls the platform and imposes rules. This approach has scalability limitations such single point of failure, server overload and network bottlenecks. In [4], the authors argue that $\mathrm{C} 2 \mathrm{C}$ commerce maps naturally onto $\mathrm{P} 2 \mathrm{P}$ systems because of its underlying interaction model of customers or peers. They advocate for the need of a decentralized system which resembles eBay but without a central authority.

P2P networks such as Kazaa ${ }^{4}$, Gnutella ${ }^{5}$, and Freenet ${ }^{6}$ scale well and attract a large number of users, but security and service guarantees become a concern which could discourage users from trading services and commodities with unknown peers. P2P systems need both proper authentication of peers and a way of guaranteeing the quality of the service.

Though our model could work on top of any P2P network, we target dynamic and decentralized $\mathrm{P} 2 \mathrm{P}$ environments such as Digital Business Ecosystems (DBE) [6]. We envisage a P2P platform relying on decentralized security and trust solutions that allows peers to securely trade and compose services. This would enable new transactions of higher risks and new

\footnotetext{
${ }^{4}$ http://www.kazaa.com/

${ }^{5}$ http://www.gnutella.com/

${ }^{6}$ http://freenetproject.org/
}

business models (e.g., dynamic service composition and virtual organizations). Our model goes beyond peer recommendations by using ratings certified by trusted peers in the system.

\section{Financial rating agencies}

In the early 1900s, industries began to require more financial capital then they could raise via the traditional relational method. Financial rating agencys rankings allowed industries to raise additional capital by allowing investors, who did not know the business venture managers, to appraise the risks and benefits of investing in the corporation. In this sense, rating agencies are independent third parties that are consulted in the course of a market transaction. Their goal is to overcome asymmetric information between both market sides (issuer and investors) by evaluating criteria according to standardized quality categories [2]. Today, three firms (Standard and Poors Corporation, Moodys Investor Services Inc., and Fitch Investors Services) dominate the financial rating market with a combined market share of roughly 94 per cent.

To arrive at a rating, there are a variety of rating models. In financial markets, the typical procedure used today is the scoring method. It relies on a well-defined set of different criteria, each of which is scored separately. The individual scores relating to the set of criteria are weighted and then added up, yielding the overall score. This score is translated in one of defined rating categories, defined as an interval on the real line that extents from minimum overall score to its maximum. Also, the Altman's Z-score is a well-known quantitative model for private firms. It is a multivariate formula that regresses historical experience on a set of accounting variables in order to determine an optimal separating function between issuer that defaulted later on and those that survived [1].

According to [9] financial rating systems are relying on a similar set of explanatory criteria which can be classified in the economic situation, the business situation, and the quality of management. The economic situation separates between the earnings (e.g. cash flows, return, etc.) and financial situation (e.g., capital structure, liquidity, financial flexibility, etc.). Criteria of the business situation describe the competition and business risk (e.g., relative market share, diversification, product mix, etc.) as well as the legal structure and legal risk. Regarding the quality of management, typical criteria in the financial rating context are organizational structure, planning and controlling, and experience.

Summing up, a financial rating system can be represent as a mathematical function:

$$
R:(\text { rating_criteria }) \rightarrow(\text { rating_category })
$$

with the rating model $R$ which assigns by weighting the rating criteria to a rating category. Most of the financial rating agencies have long had their own system of categories (also named as symbols or classes) - some using letters, others using numbers, many both - for ranking the risk of default from extremely safe to highly speculative. 


\section{Rating systems for the Internet}

A pure rating agencies model for e-business does not exist yet; however, many Web sites that independently rate software applications and Web pages based on objective criteria behave similarly to a rating agency. If an Web site passes the evaluation, it will be granted a seal from the evaluator that certifies the quality.

Tucows ${ }^{7}$, Promaxus ${ }^{8}$, and SnapFiles ${ }^{9}$ are examples of third parties that rate software applications and the Web sites dedicated to selling them. They target the quality of the application in terms of functionality and documentation and use similar criteria. Among them, Tucows uses the most standardized rating guide with four criteria with different categories: Usability, Help, documentation, and support, Program enhancements, and Reviewer's overall evaluation. The Tucows rating guide gives details about how each of the criteria is evaluated. A 56-point rating scale is used and each of the four categories is being given a different weight. The Tucows rating guide is so well defined and standardized that a third-party site, Shareware-development ${ }^{10}$ provides a Tucows rating calculator which allows software authors to analyze their program and get an idea of how it would rate on Tucows.

SiteTrust Network ${ }^{11}$ is dedicated to approved online merchants and, as opposed to the above mentioned Web sites, SiteTrust Network is concerned with the efficiency, security and legality of the online sale, but not with the quality of the products. The criteria used for evaluating a Web site is the following: Communication with the support staff, Ease of Use, Information \& Transparency, Terms \& Conditions, Security, and Legality.

WebTrust $^{12}$ targets e-commerce Web sites and a licensed WebTrust Certified Public Accountant (CPA) checks compliance with a set of principles and criteria which cover three main areas: Business practices and disclosures, transaction integrity, and information protection and privacy. Periodic reexamination is required to ensure the Web site still complies with the criteria.

\section{MODEL DESCRIPTION}

Let us start with the core terms used in the model.

- Rating agency: any peer in the system able to rate entities based on a predefined rating criteria

- Rating criteria: the criteria used by an agency to evaluate an entity.

- Rated entities: the rating agencies will rate any entity that could be described on the Web ${ }^{13}$

- Rating certificate: certificate issued to an entity to certify a rating based on certain rating criteria.

\footnotetext{
${ }^{7}$ http://tucows.totalshareware.com/about/rating.htm

${ }^{8} \mathrm{http}: / /$ www.promaxum.com/seal_of_quality.html

${ }^{9} \mathrm{http}: / /$ www.snapfiles.com/ratings.html

${ }^{10} \mathrm{http} / / / \mathrm{www}$. shareware-development.com/tucows-rating-calculator/

${ }^{11} \mathrm{http}: / / \mathrm{www}$. sitetrustnetwork.com/

${ }^{12} \mathrm{http}: / /$ www.webtrust.org/

${ }^{13} \mathrm{http}: / / \mathrm{fp} 7$. OKKAM.org
}

Rating agencies are dedicated services that could be offered by any node in the P2P network and there is no hierarchy of rating agencies. Users are free to decide which agency to trust and use and they register on a voluntary basis with one or several rating agencies. Certificates can be issued to all entities in the system: users, companies, services, data, nodes (infrastructure).

Each agency needs to clearly define the criteria on which entities are evaluated (e.g., quality standards). If an entity passes the evaluation, it will receive a certificate stating the level of quality as assessed by the agency. This certificate can be used when interacting with other entities in the network to prove the entity is trustworthy and reliable. This represents an incentive for users to register with rating agencies.

However, because of the distributed nature of the environment, the certificate will be recognized by a partner only if the partner trusts the agency. We are using a Web-of-trust approach which means that the value of the certificate is also related to the reputation and the circle of trust of the agency issuing it. Outside the social network of the institution, the certificate is not recognized. To enable inter-community trust and collaboration, we enable agencies to issue certificates based on certificates issued by one of their trusted agencies.

1) Rating certificate: In a decentralized environment, each agency defines and uses its own criteria to rate services or users. Criteria are not always immediately compatible with the criteria of another agency. In our model, a rating agency issues a rating certificate (a digitally signed document) with the used criteria and the rating for a service. First step to achieve interoperability is to adopt a standardize rating certificate representation. To this extend, we aim for a standardized security token widely acceptable which could be exchanged by parties using possibly different rating criteria. We adopted SAML [12] and its attribute assertion statements to represent different criteria and values. We chose SAML because it defines an XML standard for expressing assertions and provides protocols for exchanging them, thus achieving interoperability at message level.

2) Agency certificate: To approach interoperation between agencies using different rating model, we introduce an agency certificate provided by each agency which contains a semantic description of the rating criteria. The certificate is signed by the rating agency and available on request. This certificate contains unambiguous rating criteria defined in a human readable language. An agency certificate defines the following:

- Type of rated service: an agency describes what types of services is rating. An agency could rate one or several types of services and each the rating criteria needs to be clearly defined.

- Rating criteria: A list of criteria based on which the service is rated. For each criteria, the agency specifies how it is evaluated.

- Scoring methodology: How each of the criteria will be combined to obtain a final score.

- Rating scale: explains how the results must be interpreted. For example, there could be a 1 to 5 scale, an 
accepted/rejected scale, or continuous values. The users need to be able to understand the meaning of the rating value, i.e. how good a service is.

3) Trusted agencies certificate: Our motivation is to allow agencies in the P2P network to build their own Web of trusted rating agencies in which each agency rates according to its own criteria and interoperates with other agencies based on mutual business relations. We introduce the notion of trusted agencies certificate which is a list of the agencies with which business relations exist. This metadata certificate contains for each trusted agency a link to the agency certificate and to a certificate describing how the two agencies interoperate, i.e. the interoperation certificate. In Figure 1, Rating Agency 1 (RA1) is linked with RA2 through these certificates.

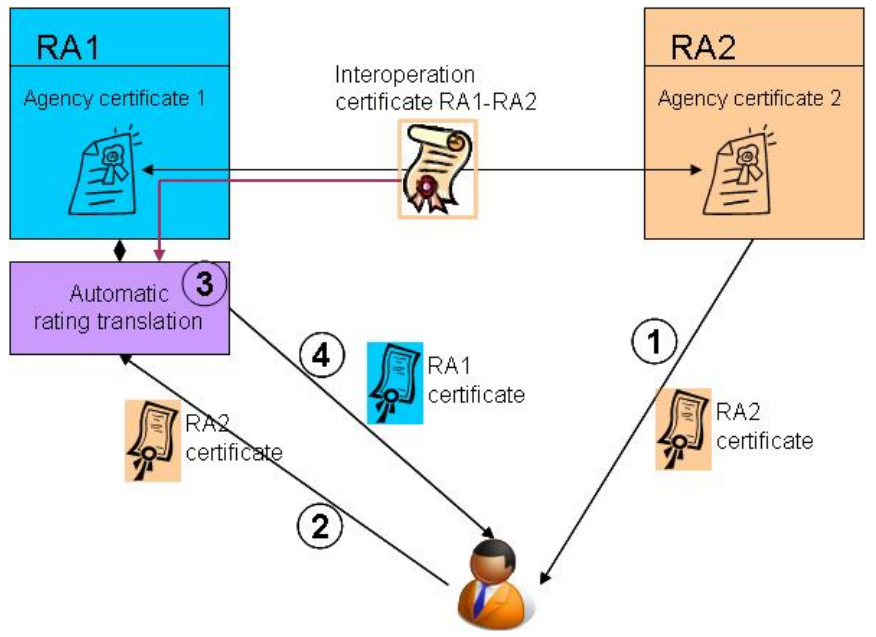

Fig. 1. Trusted rating agencies interoperation

4) Interoperation certificate and automatic certificate translation: The interoperation certificate states how the different rating criteria of two agencies relate with each other. The certificate is agreed and signed by both agencies when they establish a new trust relation. The initial translation of criteria is done manually based on the agency certificates of the two agencies. An ontology language such as OWL [11] will be used for defining the relations between the two criteria. Afterwards, the agencies can automatically translate rating certificates issued by the other one based on the translation criteria expressed in the interoperability certificate. This allows users who have a rating certificate issued by an agency to be recognized in a different domain if the agency is trusted by an agency in the other domain.

Figure 1 shows the steps necessary for a user to translate his certificate. In step 1, the user obtains a rating certificate from RA2. In step 2, the user submits a request to the automatic translation service of RA1. The translation made in step 3 is possible only because the two agencies have an agreement and have issued together an interoperation certificate RA1-RA2. In step 4, the user receives the new certificate automatically issued by RA1. If the two agencies use the same standardized rating criteria but are part of different domains, then a trans- formation of rating certificate RA2 is still necessary. The new RA1 certificate would be recognized in domains where RA2 is unknown.

Figure 2 shows an example of a $\mathrm{C} 2 \mathrm{C}$ negotiation in which automatic rating translation takes place at run time, during the execution. In step 1, while setting up the negotiation, the owner defines a list of trusted RAs (at least one) from which certificates will be accepted from during negotiation. At negotiation run time, users are allowed access to the negotiation only if they can provide a trusted certificate. In step 2, a negotiation participant requests the list of trusted RAs. If the user does not have the right certificate, it can request for a translation if a trust relation exists between an agency that already certified the user and one of the trusted RAs. In steps 3-4-5, the automated translation of rating certificates is performed analogously to Figure 1 . In step 6, the negotiation participant provides the new certificate to the negotiation engine and is granted access to the negotiation.

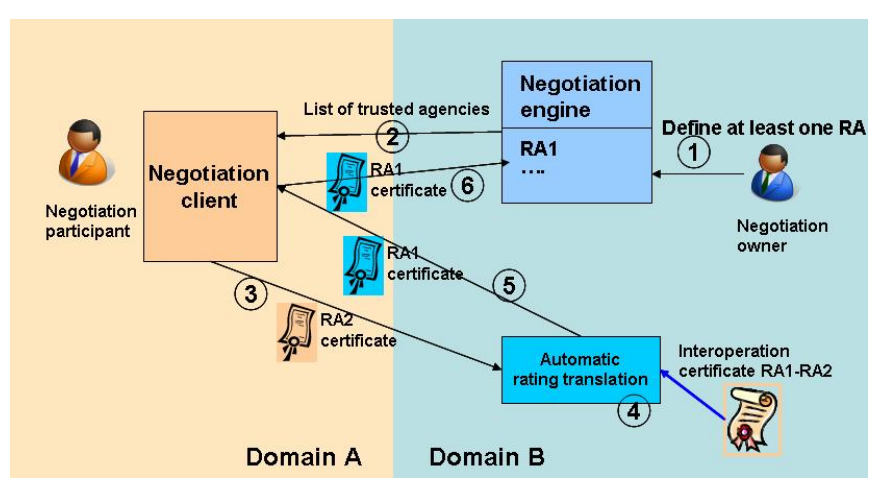

Fig. 2. Automatic rating translation at negotiation runtime

\section{Agencies Interoperation Model}

Each agency defines its own rating criteria in the form of an ontology. The keywords used to define criteria are defined in the namespace of the agency. Each agency also defines for each criteria the rating values that can be associated with it. Agencies need to define the type (e.g. percentage, 1-10 values, literals: A, B , C...), the range and the meaning of the allowed rating values: e.g. 1 can be highest rating value and mean excellent or 5 could be the highest.

We achieve interoperability between rating agencies by means of transformation of one ontology domain to another ontology domain. The transformation process is functionbased and defines mapping of criteria and value from source domain to criteria and value of destination domain. The transformation function is defined in the destination domain, i.e. it is identifiable in that domain. The notion of transformation function is generic enough to cover a wide range of possible transformation/adaptation mechanisms.

Definition 1: A criteria $\mathcal{C}^{\text {onto }}$ is an identifiable term in an agency's ontology that can be described by the following Backus-Naur form (BNF):

$$
\begin{aligned}
& <\text { letter }>::={ }^{\prime} A^{\prime} . .{ }^{\prime} Z^{\prime}\left|{ }^{\prime} a^{\prime} .{ }^{\prime} z^{\prime}\right|{ }^{\prime}{ }_{-} \\
& <\text {digit }>:=0 \mid 1 . .9
\end{aligned}
$$




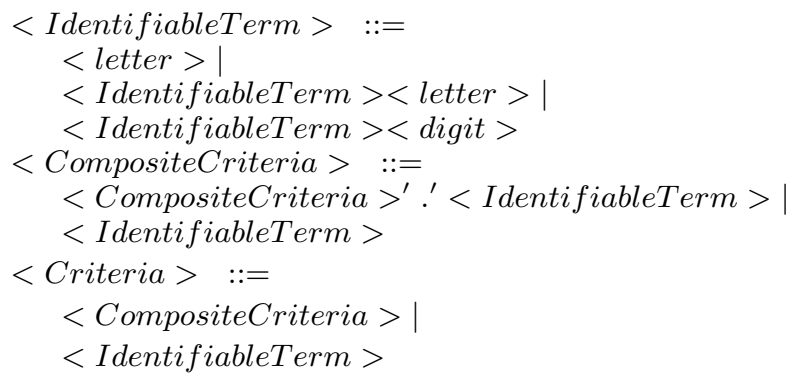

A criteria $\mathcal{C}$ can refer to just an identifiable term or can refer to a chain of category criteria to which a desired one belongs to. Let us take the example of Tucows rating guide for software applications as described in section 1.D. According to our definition and notations, the rating contains the following criteria:

Usability

Usability.Installation

Usability.Program_interface etc.

Definition 2: A rating certificate, with respect to a service of a given type, is a list of assertions that associate to a criteria a rating value, and an overall rating of the agency based on the criteria asserted in the certificate. Formally, we represent the rating certificate as $\mathcal{R C ^ { R A _ { k } }}\left(\mathcal{C}_{1}^{R A_{k}}, \ldots, \mathcal{C}_{m}^{R A_{k}}, \mathcal{V}^{R A_{k}}\right)$ where $\mathcal{C}_{i}^{R A_{k}}, 1 \leq i \leq m$ are the certified criteria, $\mathcal{V}^{R A_{k}}$ is the overall criteria and $R A_{k}$ denotes rating agency $k$ 's ontology.

A $\mathcal{C}_{i}^{R A_{k}}$ encapsulates an identifiable term (numbered $i$ ) and a rating value associated to it. We note that in some financial settings the value part may refer to a rating category or a class (see Section I.C). In the rest of the paper whenever we refer to a certified criteria we implicitly refer to a term and a value. Let us look at the following example of how SAML could encode rating criteria and its value.

Example 1: If criteria $\mathcal{C}_{i}$ is rated with value $v$, agency $R A_{k}$ will generate the following SAML attribute statement for $\mathcal{C}_{i}^{R A_{k}}$ :

$$
\begin{aligned}
& <\text { saml }: \text { AttributeStatement }> \\
& <\text { saml }: \text { Attribute } \\
& \text { NameFormat }=" h t t p: / / \text { RAk.com/attr_formats" } \\
& \text { Name }=\mathcal{C}_{i} \\
& \text { xmlns }: \text { RAk }=" \text { http }: / / \text { www.RAk.com/schema.xsd" }> \\
& \quad<\text { saml }: \text { AttributeValue } x \text { si }: \text { type }=" R A k: \text { rtype" }> \\
& \quad<\text { RAk }: \text { rtype }>v</ \text { RAk }: \text { rtype }> \\
& \quad</ \text { saml }: \text { AttributeValue }> \\
& </ \text { saml }: \text { Attribute }> \\
& </ \text { saml }: \text { AttributeStatement }>
\end{aligned}
$$

The criteria and the rating value have meaning only in the namespace of agency $R A_{k}$ where the name of the criteria and the type and range of the rating value have been defined.

Having introduced the criteria definition and technical representation next part of the section will define the syntax and semantics of the transformation process between two agencies' domains.

Definition 3: A transformation rule is a tuple of the form: $\left\langle\mathcal{C}_{1}^{R A_{k}}, \ldots, \mathcal{C}_{n}^{R A_{k}}, \mathcal{C}^{R A_{p}}, f^{R A_{p}}()\right\rangle, n \geq 1$ where $\mathcal{C}_{i}^{R A_{k}}, 1 \leq i \leq n$, are called input criteria, $\mathcal{C}^{R A_{p}}$ the output criteria and $f^{R A_{p}}()$ a transformation function.
The transformation function is an identifiable term in the domain of agency $R A_{p}$, i.e. it is defined and implemented on $R A_{p}$ 's side. Based on the above transformation rule, $R A_{p}$ computes the value of $\mathcal{C}^{R A_{p}}$ in the following way:

$$
\mathcal{C}^{R A_{p}}:=f^{R A_{p}}\left(\mathcal{C}_{1}^{R A_{k}}, \ldots, \mathcal{C}_{n}^{R A_{k}}\right)
$$

The function $f^{R A_{p}}()$ is agreed upon by the two agencies when they establish their business relation and issue an interoperation certificate. Function details and implementation are defined by $R A_{p}$ and kept on its local domain.

For $n=1$ we have a one-to-one transformation and for $n>$ 1 we have a many-to-one transformation. We handle manyto-many transformations by reducing them to a set of manyto-one transformations. When output criteria are function of input criteria then each of the output criteria can be defined as independent function of the input criteria.

Definition 4: An interoperation certificate is a set of transformation rules that define transformations of criteria and ratings between same type of services ${ }^{14}$ or possibly different types if they are comparable. Formally:

$$
\begin{aligned}
\mathcal{I}=\left\{t r_{i} \mid\right. & R A_{k} \underset{t r_{i}}{\stackrel{t r_{i}}{\longrightarrow}} R A_{p}: i \in[1 . . s], \\
& \left.R A_{k}: i \in[s+1 . . s+t]\right\}, s, t \geq 1 .
\end{aligned}
$$

An interoperation certificate contains all bilateral transformation rules necessary to transform one set of criteria (and their ratings) defined in the source domain to another set defined in the target domain and vice versa. One can also accommodate one-way transformations only in the model in case bilateral interoperability is not feasible.

Definition 5: A well-formed rating certificate with respect to an interoperation certificate $\mathcal{I}$ certifies at least those input criteria that appear in all transformation rules in $\mathcal{I}$ defined from the domain of the certificate to the domain of interoperation (destination).

The definition above gives us (the intuition of) minimal conditions for certificate issuance to guarantee an interoperability process.

An automated rating translation is done by using an interoperation certificate and a rating certificate issued by one of the partner agencies as already shown in Figure 2. Let $\mathcal{R C}^{R A_{k}}\left(\mathcal{C}_{1}^{R A_{k}}, \ldots, \mathcal{C}_{m}^{R A_{k}}, \mathcal{V}^{R A_{k}}\right)$ be a rating certificate and let $\mathcal{I}=\left\{t r_{i} \mid 1 \leq i \leq s+t\right\}$ be an interoperation certificate (rf. Definition 4) between agencies $R A_{k}$ and $R A_{p}$. We assume that the rating certificate is of a service type matching the service type of the interoperation certificate for $R A_{k}$. Algorithm 1 shows an automatic transformation process performed by agency $R A_{p}$.

The computation of the overall rating is according to an internal function specific to $R A_{p}$. We do not indicate this function in the interoperation certificate because it is not directly related to the interoperation process. One can include such information in the interoperation certificate if necessary. The new rating certificated is issued in a form of a SAML token as shown below.

\footnotetext{
${ }^{14}$ actually any identifiable item on the Web (see section 2)
} 


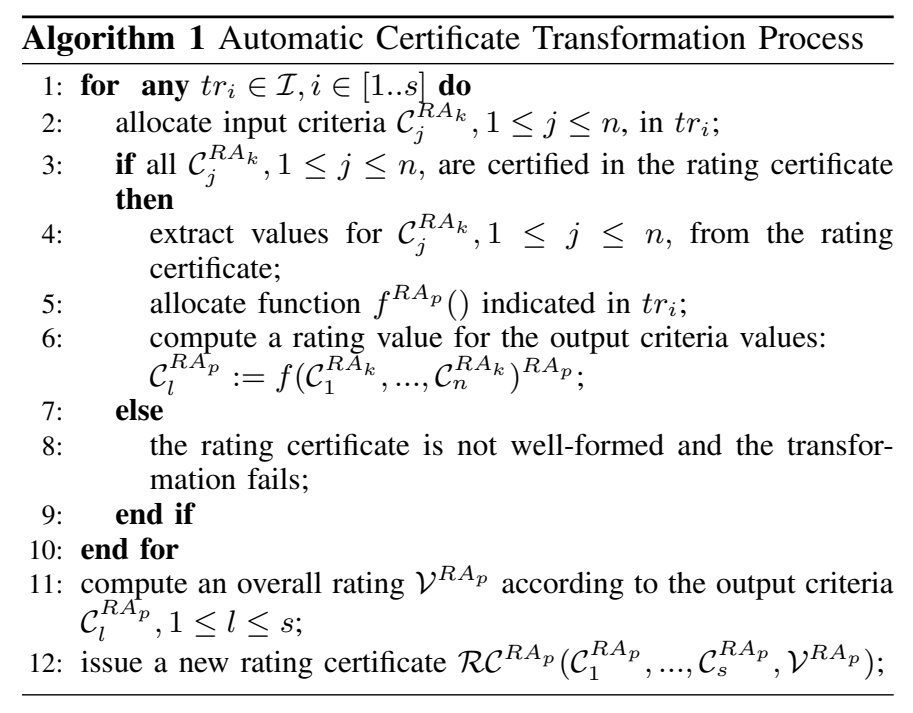

Example 2: Let us adapt the example of Tucows and SnapFiles which independently defined rating criteria for software applications (hence, for the same service type) and show how the different criteria can be transformed by using transformation rules in an interoperation certificate.

Let us assume Tucows rating certificate contains the following SAML token with assertions expressed. For simplicity, we will express the SAML assertions in an abbreviated form. The description of the meaning of rating values is indicated by $T$ for Tucow's ontology.

\section{SAML_token}

SAML_assertion(Usability.Direct_comparison ${ }^{T}$, 2) //(out of 3)

SAML_assertion(Usability.Unique_features ${ }^{T}$, 2) //( out of 2)

SAML_assertion(Overall_evaluation.Cost_vs_value $\left.{ }^{T}, 2\right) / /($ out of 3 )

)

SnapFiles, denoted with ontology $S$, uses the following rules in the interoperation certificate:

$\left\langle\right.$ Usability.Direct_comparison ${ }^{T}$, Uniqueness_and_innovation ${ }^{S}, f_{1}^{S}(\dot{)}\rangle$

$\left\langle\right.$ Overall_evaluation.Cost_vs_value ${ }^{T}$, Pricing $\left.{ }^{S}, f_{2}^{S}()\right\rangle$

where $f_{1}^{S}$ and $f_{2}^{S}$ denote two different functions of manyto-one and one-to-one transformations, respectively. The two functions implement desired computations based on the semantics of input and output criteria in the respective transformation rules. SnapFiles performs the following transformations:

Uniqueness_and_innovation $^{S}:=f_{1}^{S}(2,2)=8$;

Pricing $S:=f_{2}^{S}(2)=7$;

After performing all the transformations, SnapFiles will compute the overall rating value and issue a new rating certificate:

SAML_token(

SAML_assertion(Uniqueness_and_innovation ${ }^{S}$, 8) //out of 10 SAML_assertion(PricingS ${ }^{\text {, 7) }) / / o u t ~ o f ~} 10$

Note that the input to the functions defined by SnapFiles corresponds exactly to the type of rating criteria values defined in the ontology of Tucows.

\section{Conclusions And Future Work}

We have presented a model for interoperability between rating agencies. The model defines a manual (or semi-automated) phase of agencies agreement (setup) on interoperation certificates (transformation rules) that allow for on-the-fly automated rating interoperability in $\mathrm{P} 2 \mathrm{P}$ online transactions. Rating certificates issued by rating agencies based on predefined criteria constitute a better proof of quality than (subjective) reputation values and allow dynamic trust establishment between entities which leads to new application scenarios.

Future work is to apply the model on practical testbeds (e.g. business-to-business negotiations ${ }^{15}$ ) with a number of rating agencies and services being used and to provide an evaluation assessment on scalability of the model and overhead in run time business transactions.

\section{ACKNOWLEDGMENTS}

Hristo Koshutanski is supported by 038978 EU-MarieCurieEIF-iAccess fellowship. Mihaela Ion, Luigi Telesca, and Volker Hoyer are supported by 034744 EU-INFSO-IST ONE project.

\section{REFERENCES}

[1] E. Altman. Financial ratios, discriminant analysis and the prediction of corporate bankruptcy. Journal of Finance, pages 189-209, 1963.

[2] R. Candor and F. Packer. The credit rating industry. Journal of Fixed Income, 5(3):10-34, 1995.

[3] R. Chen and W. Yeager. Poblano: A distributed trust model for peer-topeer network, August 2001. www.jxta.org/docs/trust.pdf.

[4] A. Datta, M. Hauswirth, and K. Aberer. Beyond "web of trust": Enabling p2p e-commerce. In IEEE International Conference on E-Commerce Technology (CEC'03), 2003.

[5] P. Dewan and P. Dasgupta. Pride: peer-to-peer reputation infrastructure for decentralized environments. In WWW Alt. '04: Proceedings of the 13th international World Wide Web conference on Alternate track papers \& posters, pages 480-481, New York, NY, USA, 2004. ACM.

[6] P. Dini. Digital Business Ecosystems, chapter A scientific Foundation for Digital Ecosystems. European Commission, 2007. www.digitalecosystems.org/book/de-book2007.html.

[7] M. Ion, A. Danzi, H. Koshutanski, and L. Telesca. A peer-to-peer multidimensional trust model for digital ecosystems. In Proceedings of IEEE International Conference on Business Ecosystems and Technologies (IEEE-DEST'08). IEEE press, February 2008.

[8] S. D. Kamvar, M. T. Schlosser, and H. Garcia-Molina. The eigentrust algorithm for reputation management in $\mathrm{p} 2 \mathrm{p}$ networks. In $W W W$ '03: Proceedings of the 12th international conference on World Wide Web, New York, NY, USA, 2003. ACM Press.

[9] J.P. Krahnen and M. Weber. Generally accepted rating principles: A primer. Journal of Banking and Finance, 25:3-23, 2001.

[10] P. Massa and P. Avesani. Trust metrics on controversial users: balancing between tyranny of the majority and echo chambers. International Journal on Semantic Web and Information Systems (IJSWIS), 2007.

[11] OWL. OWL Web Ontology Language. W3C, February 2004. http://www.w3.org/TR/owl-features/.

[12] SAML. Security Assertion Markup Language (SAML), 2005. www.oasis-open.org/committees/security.

[13] A. Singh and L Liu. TrustMe: Anonymous management of trust relationships in decentralized $\mathrm{P} 2 \mathrm{P}$ systems. In $\mathrm{P} 2 \mathrm{P}$ '03: Proceedings of the 3rd International Conference on Peer-to-Peer Computing, page 142, Washington, DC, USA, 2003. IEEE Computer Society.

[14] L. Xiong and L. Liu. Reputation-based trust model for peer-topeer ecommerce communities. In IEEE Conference on E-Commerce (CEC'03), Newport Beach, USA, June 2003.

[15] R. Zhou and K. Hwang. Powertrust: A robust and scalable reputation system for trusted peer-to-peer computing. IEEE Trans. Parallel Distrib. Syst., 18(4):460-473, 2007.

\footnotetext{
${ }^{15} \mathrm{EU}$ project ONE: http://one-project.eu
} 